The following stars have been observed as III!:

$+67^{\circ} 1004$, I $564 ;+66^{\circ} 1596 ;+65^{\circ} 1069 ;+64^{\circ} 1735$.

$+63^{\circ} 1396,1740 ;+61 \% 1,270,707,741,759,1604$.

$+60^{\circ} 6,1599,2647 ;+59^{\circ} .107,2337$.

$+58: 1422,1552 ;+57: 367,1608,2091$.

+56.264 , I 747 , I $798 ;+54^{\circ}$ I 25,3 I $5,2899,3036$.

$+53^{\circ} 698 ;+52 \% 2122 ;+51.2394$.

$+50.809,1992,2098,2153,2522$.

$+49^{\circ} 1057$, I 206,$2702 ;+4892286,2314,2668,3874$.

$+47^{\circ} 5^{8} 3,904,2359,2522,2596 ;+46^{\circ} 3609$.

$+45^{\circ} .2618,2662,2927 ;+44^{\circ} 45^{6}, 560,3242$.

$+43^{\circ} .2860,2970,3072 ;+42.587,2566 ;+41.2988$.

$+40^{\circ}$ I 343,$4848 ;+39^{\circ} 325^{2}, 4457,4601,4782$.

$+38.326,4942 ;+37^{\circ} 199,4699$.

+36:4194, 4873,$5048 ;+35^{\circ} 4840 ;+34 \% 4$ I 18 .

$+33^{\circ} 4210 ;+30^{\circ} 3344,3442,3787 ;+29^{\circ} 3855,3909$.

$+28^{\circ} 3440,3626 ;+27^{\circ} 3395 ;+17^{\circ} 4535$.

$+16^{\circ} 3693,3885,3^{893} ;+15: 33^{6} 3,3511,4029,4232$.

$+\mathrm{I} 4^{\circ} 3917,4179,4377,4405 ;+13^{\circ} 3579 ;+\mathrm{II}: 4289$.

$+10^{\circ} 3596,4259 ;+9^{\circ} 3866$.

$+8.4265,4331,4349,4369,437^{6}$. only II :

The following as III, some of them being probably

$+67^{\circ} 94^{2}, 968,997 ;+66.915 ;+64^{\circ} 1656,1679$.

$+63^{\circ} 40,359$, I132, I194, I $202,1304,1360,2038$.

$+62: 12,20,207$, I 388, I 539,1826, I $892,2269,2343$.

$+61: 8,1359,1408,1427,1652$.

$+60: 50,63$, I 60, I 572, I 584, I 603, I 673, I 76 I, 2225

$+59^{\circ} 33,41,64,73,131,224,1461,1638,1716,1832$, 2 I $79,2215,2232,2285,28$ I 7 .

+58.19, I $296,1345,1437, \quad 1563,1645,1669,1698$, 2156,2 I 60,2701 .

$+57^{\circ} 1398$, I 426, I 432, I 544, I 615, I 671, I 77 I, 2026 , 2085 .

$+56: 1780,1833,1864,2024 ;+55^{\circ} 2702$.

$+54^{\circ} 1492,1869,2970,3033$.

$+53^{\circ} 55^{6}, 897,1848$, I 850 , I 910,3 I 30,3 1 $80,3242,3245$.

Tow Law, Darlington, England, I895 Jan. 7 .
$+52.624,639,655,665, \quad 703, \quad 769,843,853, \quad 1637$, 1698,3257 .

+511754, 1830, $2219 ;+50^{\circ} 764,2070,2095,2413$. $+49^{\circ} 862,2143,2256,2259,2269,2716,2729,2847$. $+48.861,953,2140,2202,2277,2576,2639,3364$.

$+47^{\circ} 784,924,953,2428,2573,2651,2949,3053,4054$.

$+46.779,853,2268,2660,2738$.

$+45^{\circ} 2764,2774,3240$.

$+44^{\circ} 272,2757,2846,4046,4087,4116,4209,4520$.

$+43^{\circ} 504,2724,3005,3025,4439,45^{23}, 4607$.

$+42: 452,2577,4572$.

$+41^{\circ} \cdot 311,430,453,2643,4668,4865,4869,4884$.

$+40^{\circ} 3295,4270,4376,4640,4676,4958,5138,5210$.

$+39^{\circ}$ 10, $2773,3104,3385,4254,4306,4379,4445$, $45^{28}, 4657,4760,4797,4814,4855,4898,4953$, $4984,5122,5195$.

$+38.120,1045,4689,4754,5013,5049$.

$+37^{\circ} 36,68,4512,4554,4852$.

$+36^{\circ}$ 1 $3,285,3^{26}, 3^{62}, 4970 ;+35^{\circ} 42$ r $8,4912$.

$+34^{\circ} 39 \mathrm{I}, 402,4136,4496 ;+33^{\circ} 4087,4276,4296,45^{80}$.

$+30^{\circ} 3^{824}, 3881 ;+29: 3^{673}, 3797,3803$.

$+28: 35^{29}, 355 \mathrm{I}, 3602$; $+27^{\circ} 3448,359 \mathrm{I}$.

$+\mathrm{I} 8: 4024,4100,4142,4865$.

$+17^{\circ} 4158,4163,4185,4297,4434,4470,4568,4607$.

$+16^{\circ} 3684,3704,3786,3792,3837,3924,4042$.

$+\mathrm{r} 5^{\circ} 3354,3408,3549,3627,3693,3745,3985,4124$, 4152.

+I 4:3931, 3972, 4I 7 I, 4213,4280, 4360.

+ $3^{\circ} \cdot 3617,3702,3^{842,3887,4548 ;+11: 3735 . ~}$

$+10^{\circ} 3727,4077 ;+9^{\circ} 4023,4064,4071 ;+8.4155$.

Herr F. Krüger has called my attention to several errors in A. N. 3200 , which have probably arisen in copying:

Es.Birm. 740 is BD. $+58.698 ; 74 \mathrm{I}$ is $56: 88 \mathrm{I} ; 753$ is $54.1010 ; 762$ is $51 \% 1228 ; 764$ is $57^{\circ} .999 ; 779$ is $50^{\circ} \mathrm{I} 600 ; 788$ is $42: 2095 ; 789 \alpha \mathrm{Ir}^{\mathrm{h}} 5^{\mathrm{m}} 6 ; 824$ is $52: 1903$; 830 is $52: 2159$. - In A.N. 3232896 is $40: 4051 ; 964$ is $46.4187 ; 922$ is $53^{\circ} \cdot 258 \mathrm{I}$. - In the third list of A. N. $323^{2}+66: 1837$ should be $+66: 873$.

\section{T. E. Espin.}

\title{
Beobachtungen von Cometen und Planeten
}

angestellt a uf der Kgl. Sternwarte in Berlin.

Instrument: Aequatoreal, $4 \stackrel{\mathrm{m}}{3}$ Focaldistanz, $244 \mathrm{~mm}$ Oeffnung. Fadenmikrometer.

\begin{tabular}{|c|c|c|c|c|c|c|c|c|c|c|c|c|}
\hline Datum & M.Z.Berlin & $\Delta a$ & $\Delta \delta$ & Vgl. & Gr. & & $\alpha$ app. & $\log p \cdot \Delta$ & $\delta$ app. & $\log p \cdot \Delta$ & Red. ad 1. app. & $*$ \\
\hline \multicolumn{5}{|l|}{ I 894} & \multicolumn{8}{|c|}{ Comet 1894 II. } \\
\hline Mai 6 & $9^{\mathrm{h}} 44^{\mathrm{m}} 42^{\mathrm{s}}$ & $-0^{m} 3^{s} \cdot 5 \mathrm{I} \mid$ & $+2^{\prime} \quad 3^{\prime \prime} 4$ & $15 \cdot 5$ & - & $8^{\mathrm{h}}$ & $48^{\mathrm{m}} 24^{\mathrm{s}} \cdot 39$ & 8.945 & $+4^{\circ} 4^{\prime} 25^{\prime \prime} 0$ & 0.822 & $+0.74-1.12$ & $\mathbf{I}$ \\
\hline 9 & $952 \quad 30$ & to 36.80 & +429.5 & I 8.6 & - & 9 & I 437.84 & 8.785 & +131452.2 & $0.75^{\circ}$ & $+0.96+1.0$ & 2 \\
\hline 9 & Io I 544 & to 42.96 & -445.2 & I 8.6 & - & 9 & I 445.74 & $8.99 \mathrm{I}$ & +131730.1 & $0.75 \mathrm{I}$ & $+0.9^{6}+1.0$ & 3 \\
\hline I 9 & IO 2450 & to 56.33 & $\begin{array}{ll}-4 & 9.0\end{array}$ & 18.6 & - & 10 & $16 \quad 4.35$ & 8.204 & +303751.6 & 0.517 & $+1.47+4.8$ & 4 \\
\hline
\end{tabular}

1894

(80) Sappho.

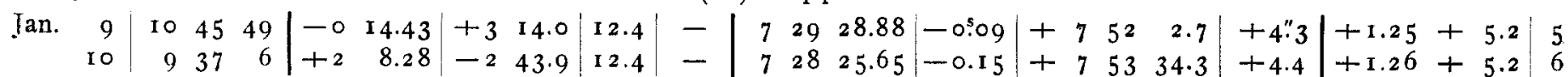




\begin{tabular}{l|l|l|l|l|l|l|l|l|l|l|}
\hline Datum & M.Z. Berlin & $\Delta \alpha$ & $\Delta \delta$ & Vgl. & Gr. & $\alpha$ app. & Par. & $\delta$ app. & Par. & Red. ad l. app. \\
\hline
\end{tabular}

I $894 \quad$ (86) Semele.

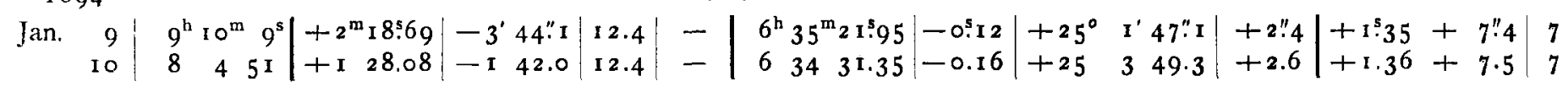

(181) Eucharis.

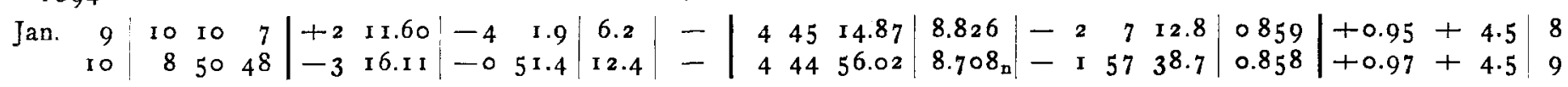

1893

(190) Ismene.

Sept. 14 10 $1022 \mid-0$ 1 $7.00|-035.8| 15.5 \mid$ I $3.0 \mid 225$ I $30.89|-0.03|-52313.9|+2.3|+3.13+16.4 \mid 10$ 1893

(326) Tamara.

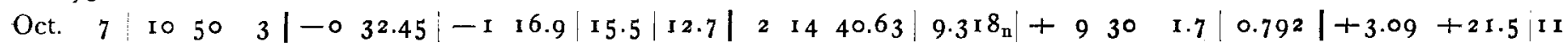

Mittlere Oerter der Vergleichsterne, bezogen auf den jedesmaligen Jahresanfang.

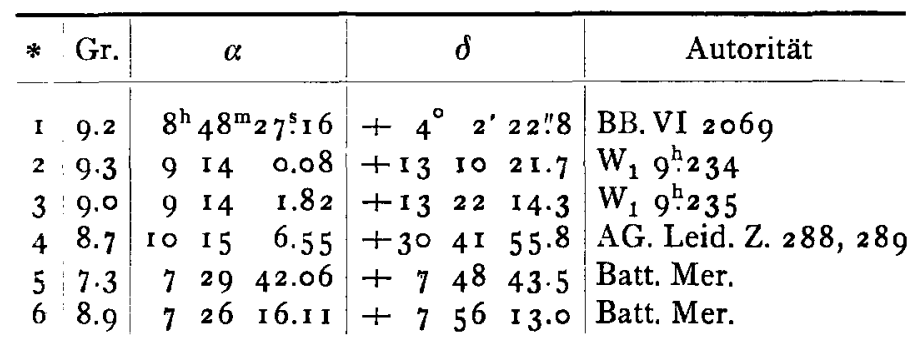

\begin{tabular}{|c|c|c|c|c|}
\hline * & Gr. & $\alpha$ & $\delta$ & Autorität \\
\hline 7 & 9.0 & $6^{\mathrm{h}} 33^{\mathrm{m}} \quad 1^{\mathrm{s}} \cdot 9 \mathrm{I}$ & $+25^{\circ} 5^{\prime} 23^{\prime \prime} 8$ & AG. Berl. Z. 197,377 \\
\hline 8 & 8.6 & $\begin{array}{lll}4 & 43 & 2.32\end{array}$ & $\begin{array}{lll}-2 & 3 & 15.4\end{array}$ & Batt. Mer. \\
\hline 9 & 8.7 & 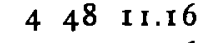 & - I 5651.8 & Batt. Mer. \\
\hline Io & 6.5 & $\begin{array}{lll}22 & 51 & 44.76\end{array}$ & -52254.5 & $\begin{array}{r}1 / 3\left(\text { Y. } 10379+\mathrm{Gl}_{1}\right. \\
\left.6006+\mathrm{Gl}_{2} 2006\right)\end{array}$ \\
\hline I I & 8.3 & $\begin{array}{lll}2 & 15 & 9.99\end{array}$ & +93057.1 & Batt. Mer. \\
\hline
\end{tabular}

Vergleichung der Beobachtungen mit Ephemeriden $(B-R)$ :

\begin{tabular}{l|l|l}
\hline $\mathrm{I} 894$ & $\Delta \alpha$ & $\Delta \delta$ \\
\hline
\end{tabular}

(8o) Sappho.

[Berl. Jahrb. I 895.]

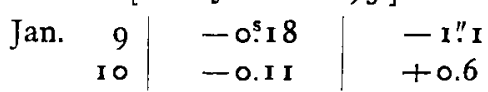

\begin{tabular}{l|l|l}
\hline $\mathrm{1} 894$ & $\Delta \alpha$ & $\Delta \delta$ \\
\hline
\end{tabular}

(86) Semele.

[Berl. Jahrb. I 895.]

Jan. \begin{tabular}{r|r|r}
9 & +1.92 & +0.4 \\
10 & +1.92 & -0.5
\end{tabular}

\begin{tabular}{c|c|c}
\hline 1893 & $\Delta \alpha$ & $\Delta \delta$ \\
\hline \multicolumn{3}{c}{ (190) Ismene. }
\end{tabular}

[Berl. Jahrb. I 895.]

Sept. $14|+0.46|-r .4$

Die mit »Batt. Mer.« bezeichneten Sterne hat Herr Dr. Battermann bestimmt. Ausserdem habe ich ihm die Neubestimmung der folgenden Sterne zu verdanken.

Vergleichsterne für die Beobachtungen von Cometen und kleinen Planeten in den Astr. Nachr. Bd. 133, p. 57-62,

neubestimmt am grossen Meridiankreise der Königl. Sternwarte in Berlin von Dr. H. Battermann.

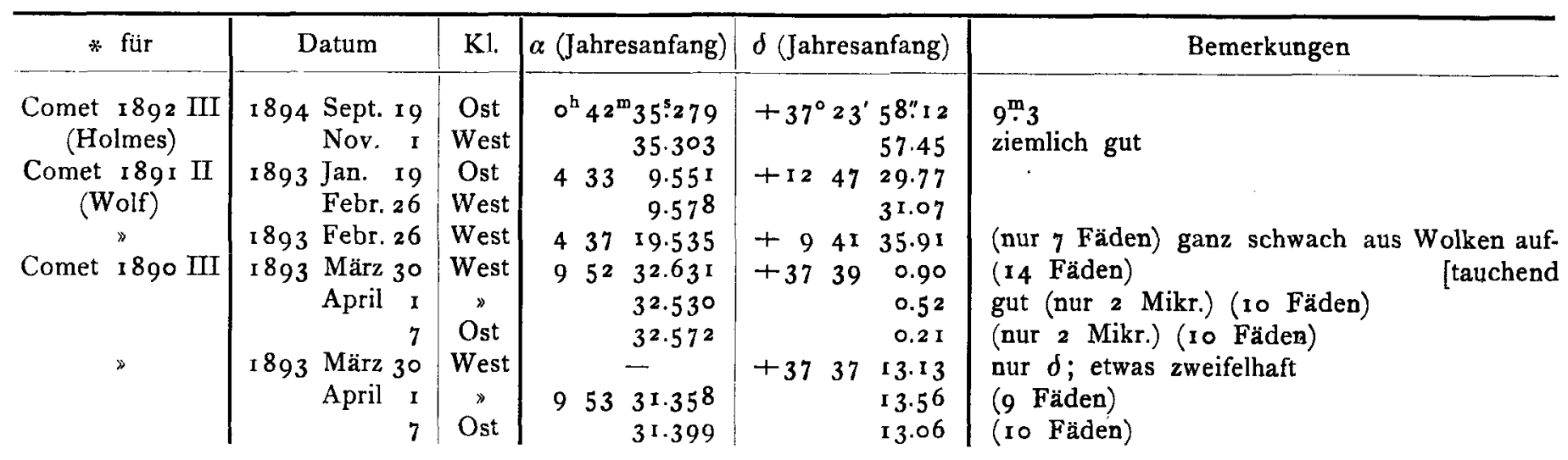




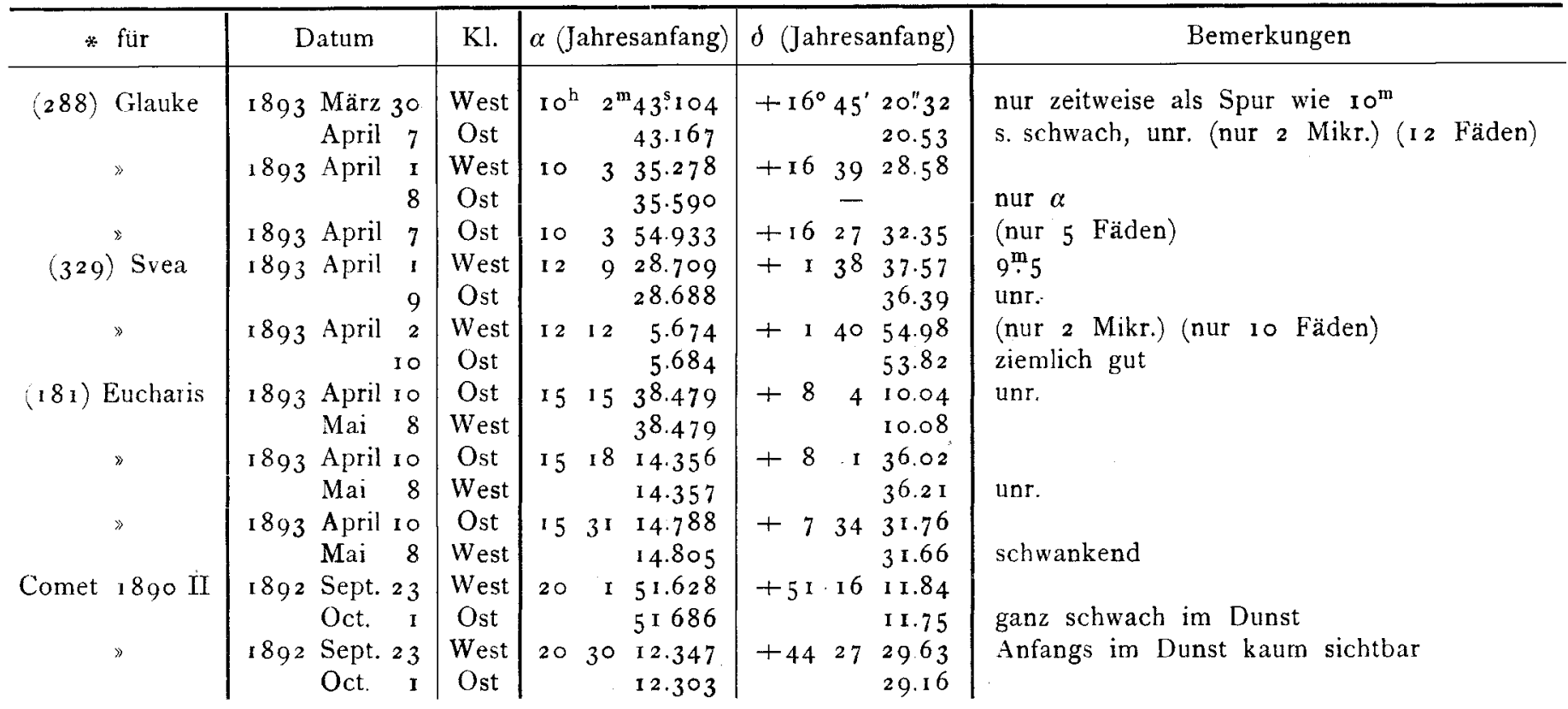

Berlin, Kgl. Sternwarte, 1895 Februar.

V. Knorre

Beobachtung der Plejadenbedeckung durch den Mond am 7. Januar 1895 auf der k. k. Sternwarte in Prag.

(Mitgetheilt von dem Director, Prof. Dr. L. Weinek.)

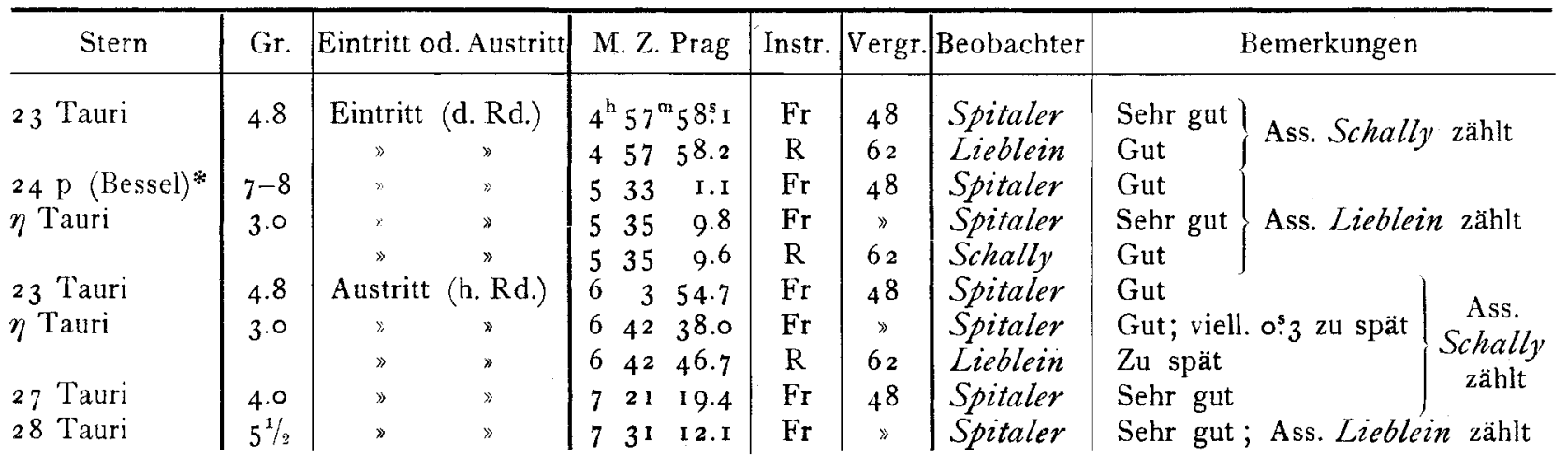

Fr = Fraunhofer'sches Fernrohr, Oeffnung $97.6 \mathrm{~mm}$; transportabel.

$\mathrm{R}=$ Reinfelder'sches Fernrohr, Oeffnung $108.6 \mathrm{~mm}$; transportabel.

Luft etwas dunstig, am Horizont Nebel. Die schwächeren Sterne waren, zumal wenn kleiner Mondhof auftrat, schwer zu sehen; in der Nähe des hellen Mondrandes wurden sie ganz unsichtbar.

Die geringe Anzahl der am Reinfelder'schen ${ }_{4}$ Zöller erhaltenen Sterne erklärt sich wesentlich aus dem Um. stande, dass das Objectiv dieses Instruments mit der Zeit blind geworden ist, so dass auch bei klarer Luft im Gesichtsfelde desselben der Himmelsgrund nicht dunkel, sondern milchig erscheint. Es liegt die Absicht vor, dieses Objectiv alsbald behufs gründlicher Reinigung nach München zu senden.

Prag 1895 Jan. 22.

\section{Weinek.}

\footnotetext{
* Der Austritt dieses Sterns wurde nicht gesehen; der Stern wurde erst wieder nach dem Austritt von $\eta$ Tauri wahrgenommen.
} 